\title{
Antivascular Treatment of Solid Melanoma Tumors with Bacteriochlorophyll-serine-based Photodynamic Therapy"
}

\author{
Judith Zilberstein ${ }^{1,3}$, Smadar Schreiber ${ }^{1,3}$, Monique C. W. M. Bloemers', Peter Bendel ${ }^{2}$, Michal Neeman', \\ Edna Schechtman ${ }^{4}$, Fortune Kohen ${ }^{1}$, Avigdor Scherz $^{3}$ and Yoram Salomon ${ }^{\star 1}$ \\ The Departments of ${ }^{1}$ Biological Regulation, ${ }^{2}$ Chemical Services and ${ }^{3}$ Plant Sciences, The Weizmann Institute of Science, \\ Rehovot, Israel and \\ ${ }^{4}$ The Department of Industrial Engineering and Management, Ben Gurion University of the Negev, Beer Sheva, Israel
}

Received 21 June 2000; accepted 14 December 2000

\section{ABSTRACT}

We describe here a strategy for photodynamic eradication of solid melanoma tumors that is based on photoinduced vascular destruction. The suggested protocol relies on synchronizing illumination with maximal circulating drug concentration in the tumor vasculature attained within the first minute after administrating the sensitizer. This differs from conventional photodynamic therapy (PDT) of tumors where illumination coincides with a maximal concentration differential of sensitizer in favor of the tumor, relative to the normal surrounding tissue. This time window is often achieved after a delay (3-48 h) following sensitizer administration. We used a novel photosensitizer, bacteriochlorophyll-serine (BchlSer), which is water soluble, highly toxic upon illumination in the near-infrared $\left(\lambda_{\max } 765-780 \mathrm{~nm}\right)$ and clears from the circulation in less than $24 \mathrm{~h}$. Nude CD1 mice bearing malignant $M 2 R$ melanotic melanoma xenografts $\left(76-212 \mathrm{~mm}^{3}\right)$ received a single complete treatment session. Massive vascular damage was already apparent $1 \mathrm{~h}$ after treatment. Changes in vascular permeability were observed in vivo using contrast-enhanced magnetic resonance imaging (MRI), with the contrast reagent GdDTPA, by shortening spin-spin relaxation time because of hemorrhage formation and by determination of vascular macromolecular leakage. Twenty-four hours after treatment a complete arrest of vascular perfusion was observed by Gd-DTPA-enhanced MRI. Histopathology performed at the same time confirmed primary vascular damage with occlusive thrombi, hemorrhage and tumor necrosis. The success rate of cure of over $80 \%$ with BchlSer indicates the benefits of the short and effective treatment protocol. Combining the sensitizer administration and illumination steps into one treatment session (30 min) suggests a clear advantage for future PDT of solid tumors.

\footnotetext{
IIPosted on the website on 20 December 2000

*To whom correspondence should be addressed at: Department of Biological Regulation, The Weizmann Institute of Science, Rehovot 76100, Israel. Fax: 972-8-934-4116; e-mail: yoram.salomon@weizmann.ac.il

(C) 2001 American Society for Photobiology 0031-8655/00 $\$ 5.00+0.00$
}

\section{INTRODUCTION}

Most clinical strategies for cancer therapy aim at direct killing of the malignant tumor cells. This approach has several drawbacks: (1) the delivery of toxic agents including chemotherapeutic drugs or macromolecular-targeted toxins into the tumor is limited by vascular barriers and diffusion within the interstitial space; and (2) the rate of successful treatment (i.e. chemotherapy and radiotherapy) (1) and especially photodynamic therapy (PDT) $\dagger$ are often oxygen-dependent (2). Thus, cells that reside farther from capillaries and, in particular, within hypoxic tumor domains, may escape poisoning $(3,4)$ resulting in a higher risk of tumor relapse. However, an alternative approach to tumor therapy is to induce cell necrosis by occlusion of the tumor blood supply. Indeed, a significant percentage of tumor-implanted mice were cured after intravenous (i.v.) injection of the Streptococcus B toxin, CM101, which selectively destroys neovasculature (5) or with Class II ricin A chain immunotoxin (6). Tumor treatment with a truncated form of tissue-factor led to complete tumor regression in $38 \%$ of the treated mice (7). In addition, control of tumors by vascular suppression (8) and the successful eradication of tumors using antiangiogenic factors (9) have been reported recently.

Although vascular damage has also been described in photodynamically treated tumors $(10-16)$ it is not the primary target of clinically applied PDT protocols in oncology. Furthermore, with photofrin ${ }^{\varpi}$ there have been indications that, rather than improving the success rate of cure, occlusion of blood capillaries with the consequent slowdown in perfusion actually reduced treatment efficacy because of oxygen deprivation (17). In recent years the importance of direct pho-

\footnotetext{
$\dagger$ Abbreviations: Bchl-Ser, bacteriochlorophyll-serine; Bchl-Ser-Ox, oxidized Bchl-Ser; Bchl $a$, bacteriochlorophyll $a$; BPD, benzoporphyrin derivative; Bphe-Ser, bacteriopheophitin-Ser; EuDTPA, Europium-diethylenetriamin-pentaacetate; Eu-IgG, Europium immunoglobulin G; Gd-DTPA, gadolinium-diethylenetriamin-pentaacetate; HPD, hematoporphyrin derivative; IgG, immunoglobulin G; i.p., intraperitoneal; i.v., intravenous; MRI, magnetic resonance imaging; NIR, near-infrared; PBS, phosphate buffered saline; PDT, photodynamic therapy; ROI, region of in terest; RF, radio frequency; $\mathrm{SD}$, spin density; $\mathrm{T}_{1}$, spin-lattice relaxation time; $\mathrm{T}_{2}$, spin-spin relaxation time; TE, time of echo; $\mathrm{TR}$, time of repetition
} 
todynamic damage to the tumor vasculature has been realized as an effective strategy in therapy of solid tumors $(18,19)$. Moreover, in PDT of choroidal neovascularization associated with age-related macular degeneration, the blood vessels are the only target of the treatment (20).

In light of these reports we set out to develop a PDT protocol that would result in the obstruction of the vascular bed of solid tumors. Applying this methodology was expected to destroy the malignant tumor component even without photodynamic destruction of each individual tumor cell. Aiming at relatively large tumors $\left(\geq 100 \mathrm{~mm}^{3}\right)$ we looked for photosensitizers that are highly phototoxic and (1) can be excited by near-infrared (NIR) radiation, where the light field penetrates deeply into animal tissues; (2) are watersoluble and therefore rapidly attain a high concentration within the tumor vasculature; (3) clear fast from the circulation; and (4) can diffuse easily within the tumor interstitial space, which is mostly hydrophilic.

The photosynthetic pigment, bacteriochlorophyll $a$ (Bchl $a$ ), strongly absorbs in the NIR. Native Bchl $a$ and its hydrophobic derivatives such as Bchl $a$ methyl ester or bacteriochlorin were found to induce vascular occlusion if illuminated shortly after administration (16), but their low solubility and overall efficiency was inferior to other, shorter wavelength-absorbing PDT reagents (e.g. photofrin $\mathrm{II}^{\circledast}$ or Chlorine e6) $(16,21-23)$ with the exception of melanotic melanoma. The latter tumors are not responsive to PDT with sensitizers that are excited by visible light since endogenous melanin, which extensively absorbs in the visible, strongly attenuates the incident radiation (24). However, PDT of shallow melanoma tumors with bacteriochlorin $a$ methyl ester was found to be effective (25).

Bacteriochlorophyll-serine (Bchl-Ser), a novel conjugate of Bchla, synthesized in our laboratory (26) seems to be an appropriate candidate for tumor therapy. Specifically, BchlSer has strong absorption at $765-780 \mathrm{~nm}$, is $\sim 200$ times more phototoxic than hematoporphyrin derivatives (HPD) in cell cultures $\left(\mathrm{LD}_{50}=5 \times 10^{-8} \mathrm{M}\right.$ in $\mathrm{M} 2 \mathrm{R}$ cell cultures), rapidly clears from the animal body (16 h) (27) and is watersoluble. Bchl-Ser was found to be phototoxic to bacteria, and immunoglobulin G (IgG) conjugates of Bchl were demonstrated to deliver phototoxicity to Staphylococcus aureus by binding to protein A residues on the bacterial cell wall (28).

Preliminary studies indicated a complete regression of $\mathrm{M} 2 \mathrm{R}$ tumors implanted in mice that were illuminated immediately after Bchl-Ser was administered. At this time the drug concentration in the blood is highest. Consequently, the tumor vasculature becomes exposed to maximal photodynamic activity (29-33). These treatment conditions rapidly induce the arrest of the oxygen supply to the Bchl-Ser-treated tumor, as we previously determined using a tissue-inserted oxygen microsensor (32).

In the present report we describe several parameters of tumor response and the rate of animal survival after applying this treatment strategy in which sensitizer administration is immediately followed by illumination. Here we demonstrate that the tumor vasculature is the primary target for BchlSer-based PDT with this protocol.

\section{MATERIALS AND METHODS}

Photosensitizer. Bchl-Ser was prepared from the natural pigment Bchl $a$ as previously described (34). The sensitizer has a major absorption band at $780 \mathrm{~nm}, \epsilon_{0}=7 \times 10^{4}(27)$. Bchl-Ser was kept dry under argon at $-20^{\circ} \mathrm{C}$ until use.

Animals. CD1 male nude mice (30-35 g) were used in all studies. Studies were performed according to Institute Animal Welfare regulations.

Tumor model. Monolayers of M2R cells were maintained as previously described (35). For tumor implantation the cells were scraped, suspended in normal saline $\left(1 \times 10^{6}\right.$ cells $\left./ 0.025 \mathrm{~mL}\right)$ and subcutaneously injected on the back of the mice as previously described (36). In about 2-3 weeks well-defined dark solid nonmetastatic melanotic tumors developed, with an average diameter of 7 $9 \mathrm{~mm}$ and a tumor thickness of 3-5 $\mathrm{mm}$. The life expectancy of this model was nearly 60 days, and a complete cure was defined as animal survival $>100$ days without recurrence. Tumor burden (clinical symptoms of pain/stress/water, food intake) was avoided by euthanizing the mice with $\mathrm{CO}_{2}$ and scoring animal demise. Animal survival curves were plotted according to Kaplan and Meier (37). For studying macromolecular leakage we implanted two tumors, one on each flank. One received complete treatment and the other served as a contralateral, dark control.

Anesthesia. Mice were anesthetized by intraperitoneal (i.p.) injection of Ketamine (Rhone Merieux, Lyon, France) $(5 \mathrm{mg} / \mathrm{kg})$ and Xylazine (Bayer, Leverkusen, Germany) $(1 \mathrm{mg} / \mathrm{kg}, 40 \mu \mathrm{L})$. This dose was found sufficient to keep the animal anesthetized for about $30-40 \mathrm{~min}$. Additional injections of $10-20 \mu \mathrm{L}$ allowed anesthesia for up to $8 \mathrm{~h}$.

Determination of Bchl-Ser concentrations in mouse blood. BchlSer $(20 \mathrm{mg} / \mathrm{kg})$ was i.v. injected into anesthetized animals that were kept in the dark until sacrificed at the indicated times. The control group was not injected. The mice were sacrificed by a cut through the carotid arteries and the blood was collected into preweighed test tubes, immediately frozen on dry ice and stored at $-20^{\circ} \mathrm{C}$ until analyzed. Bchl-Ser concentrations were determined fluorometrically in the acetone extracts of the blood samples as described previously (27). Bchl-Ser and derivatives content was expressed as micrograms per milliliter blood.

Photodynamic treatment. Bchl-Ser administration was performed by an i.v. injection of $20 \mathrm{mg} / \mathrm{kg}$ of Bchl-Ser in $0.2 \mathrm{~mL}$ alcohol $(95 \%)$ :saline 1:10. The drug dose used in these experiments was selected from preliminary data as the minimal effective dose inducing severe necrosis in the treated tumors. The anesthetized mouse was placed in a home-built chamber and the tumor $(7-9 \mathrm{~mm})$ was immediately illuminated (light spot diameter $1.2 \mathrm{~cm}$ ) for $30 \mathrm{~min}$. The light source, Versa-light xenon lamp (Medic-Light, Haifa, Israel) equipped with a bundle of optic fibers had a total output of $150 \mathrm{~mW}$ with a spectral window of $580-750 \mathrm{~nm}$. The treatment intensities were $60-100 \mathrm{~mW} / \mathrm{cm}^{2}$ equivalent to $108-180 \mathrm{~J} / \mathrm{cm}^{2}$ at 30 min exposure.

Determination of vascular leakage of Eu-IgG. To determine PDTinduced vascular leakage, we measured the release of fluorescently labeled mouse IgG (Sigma Chemical Co., St. Louis, MO) labeled with Europium-diethylenetriamin-pentaacetate (Eu-DTPA) Europium immunoglobulin $\mathrm{G}$ (Europium-IgG) (38) from the blood stream to the tumor tissue. Mice implanted with two tumors, one on each flank were i.v.-injected with Bchl-Ser $(15 \mathrm{mg} / \mathrm{kg})$; only one tumor was illuminated while the tumor on the contralateral side was kept in the dark (dark control). Eu-IgG was diluted in phosphate buffered saline (PBS) and i.v.-injected $1 \mathrm{~h}$ after PDT. Twenty minutes later the mice were sacrificed and the tumors (control and treated) as well as the kidneys (both sides) were removed for fluorescence analysis. The tissues were subsequently homogenized in PBS, centrifuged with an airfuge (100000 $g$ for $10 \mathrm{~min}$ ) and the supernatant analyzed for fluorescence in enhancement solution (Wallac Oy EG\&G, Turku, Finland). Time-resolved fluorescence was determined by using an LKB-Wallac, Arcus 1230 fluorimeter.

In vivo MR imaging. MRI was performed on a 4.7 Tesla spectrometer with a horizontal bore magnet (Bruker-"Biospec", Germany). Briefly, the anesthetized mouse was placed with the tumor positioned above the center of a $2 \mathrm{~cm}$ surface radio frequency (RF) coil. 


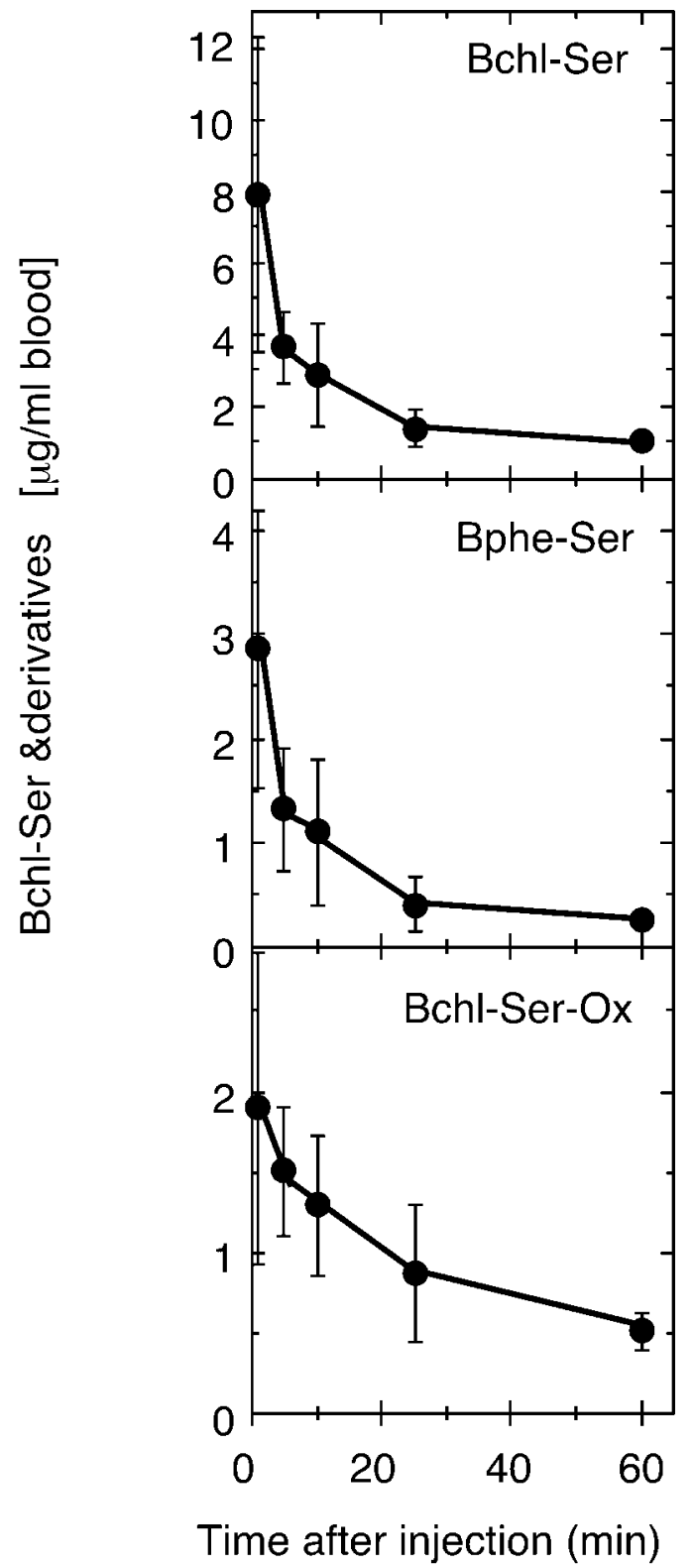

Figure 1. Bchl-Ser levels in the mouse blood during the first hour after injection. Anesthetized CD1 nude mice were i.v.-injected with Bchl-Ser $20 \mathrm{mg} / \mathrm{kg}$ (five mice/time point) and kept in the dark until sacrificed with $\mathrm{CO}_{2}$. The control group was not injected. The blood concentrations of Bchl-Ser, Bphe-Ser and Bchl-Ser-Ox are presented as a function of time. All other details are as described under "Materials and Methods."

Contrast-enhanced MRI was recorded to determine tumor vessel integrity by following the kinetics of the signal enhancement by gadolinium-diethylenetriamin-pentaacetate (Gd-DTPA) after an i.v. injection of $0.1 \mathrm{~mL}$ Gd-DTPA $(0.5 \mathrm{mmol} / \mathrm{kg})$ (39). Serial spin-lattice relaxation time $\left(T_{1}\right)$-weighted gradient echo images (time of repetition $[\mathrm{TR}]=30 \mathrm{~ms}$, time of echo $[\mathrm{TE}]=10.5 \mathrm{~ms}$, slice thickness $=1 \mathrm{~mm}, 256 \times 256$ pixels with a plane resolution of $110 \mu \mathrm{m}$ ) were acquired every $60 \mathrm{~s}$ over a $20 \mathrm{~min}$ period after the injection. The injection was performed after collecting 2-3 baseline images (time zero). Signal enhancement time curves were generated from regions of interest as indicated in the difference images as previously described by us (39).

Spin-spin relaxation time $\left(\mathrm{T}_{2}\right)$-weighted images were acquired using a cylindrical transmitter and an active RF decoupled surface receiver coil, $2 \mathrm{~cm}$ in diameter. $\mathrm{T}_{2}$ maps were generated from mul-
Table 1. Peak blood levels of sensitizer one minute after i.v. injection

\begin{tabular}{lcccc}
\hline Sensitizer & $\begin{array}{c}\text { Peak at } \\
(\mathrm{min})\end{array}$ & $\begin{array}{c}\text { Peak level } \mu \mathrm{g} / \mathrm{g} \\
\pm \mathrm{SD} \mathrm{n}=5\end{array}$ & $\begin{array}{c}\mathrm{t}_{1 / 2}(\mathrm{I}) \\
(\mathrm{min})\end{array}$ & $\begin{array}{c}\mathrm{t}_{1 / 2}(\mathrm{II}) \\
(\mathrm{min})\end{array}$ \\
\hline Bchl-Ser & 1 & $7.88 \pm 4.39$ & 6 & 73 \\
Bphe-Ser & 1 & $2.86 \pm 1.34$ & 7 & 54 \\
Bchl-Ser-Ox & 1 & $1.90 \pm 0.97$ & 22 & - \\
\hline
\end{tabular}

tiecho pulse sequences (0-90 ms) with echo intervals of $15 \mathrm{~ms}$, a repetition time of $1273 \mathrm{~ms}$, a slice thickness of $1 \mathrm{~mm}$ and a field of view of $4 \mathrm{~cm}$ over a $256 \times 256$ matrix, resulting in a spatial resolution of $156 \mu \mathrm{m}$. Serial $\mathrm{T}_{2}$-weighted spin echo images were acquired over a $20 \mathrm{~min}$ period after an i.v. injection of Gd-DTPA (segmented rapid acquisition relaxation enhanced (RARE), eight echoes per excitation with an effective TE of $77 \mathrm{~ms}$ ). The field of view was $4 \mathrm{~cm}$ over a $256 \times 128$ matrix.

Data and image processing. Data collected by the Biospec spectrometer were analyzed by using IMAGE software (New Methods Res., St. Louis, MO) with a personal Iris workstation or with Paravision software (Bruker) on an Indigo-2 workstation (Silicon Graphics, Mountain View, CA). $T_{2}$ maps were generated by pixelby-pixel single exponential best fit. Animal survival curves were compared using log rank and Wilcoxon tests according to (40) and the LIFETEST procedure of SAS (SAS ${ }^{\circledast}$ User's Guide: statistics, SAS Institute Inc., Cary, NC). The degree of PDT-induced macromolecular leakage into PD-treated tumor was analyzed in comparison to contralateral untreated tumors by statistical analysis of the ratio of accumulation of i.v.-injected Eu-IgG using Wilcoxon signed rank test (41).

Histopathology. For histopathological examination of the PDT results the mice were sacrificed $24 \mathrm{~h}$ after treatment (by cervical dislocation) and the tumors removed and placed in Buene's fixative for $24 \mathrm{~h}$. The tumors were then processed, paraffin-embedded, sectioned $(2-3 \mu \mathrm{m})$ and stained with hematoxylin/eosin without or with lightgreen staining. Light microscopy was performed using Optiphot-2 (Nikon, Japan).

\section{RESULTS}

\section{Bchl-Ser blood level determination}

The rapid clearance of Bchl-Ser after i.p. injection (27) suggested that the intravenous route should be preferred, since maximal sensitizer concentrations in the blood will be reached within a shorter time. We examined the blood BchlSer levels in the treated mice during the first hour after i.v. injection (Fig. 1). We found that the highest blood levels $(7.9 \pm 4.4 \mu \mathrm{g}$ Bchl-Ser/mL blood) are attained within the first minute after injection (the shortest time sampled) and sharply decline thereafter $\left(t_{1 / 2}=6 \mathrm{~min}\right)$ to a level of $1 \mu \mathrm{g}$ / $\mathrm{mL}$ blood within $60 \mathrm{~min}$. Similar results were obtained upon analysis of the degradation products bacteriopheophitin-Ser (Bphe-Ser) and oxidized Bchl-Ser (Bchl-Ser-Ox), which are also photodynamically active (Table 1, Fig. 1). These results clearly linked the blood levels of Bchl-Ser with optimal timing of the tumor response to PDT, suggesting that illumination shall follow Bchl-Ser injection without delay.

\section{PDT of solid melanoma tumors: animal survival}

M2R melanoma cells $\left(10^{6}\right)$ inoculated subcutaneously into nude mice developed solid dark tumors. Within 14-21 days, when the tumors reached a diameter of about 7-9 $\mathrm{mm}(\sim 76-$ $212 \mathrm{~mm}^{3}$ ), PD treatment was performed. Untreated tumorbearing animals had a mean survival time of 50.7 days after 


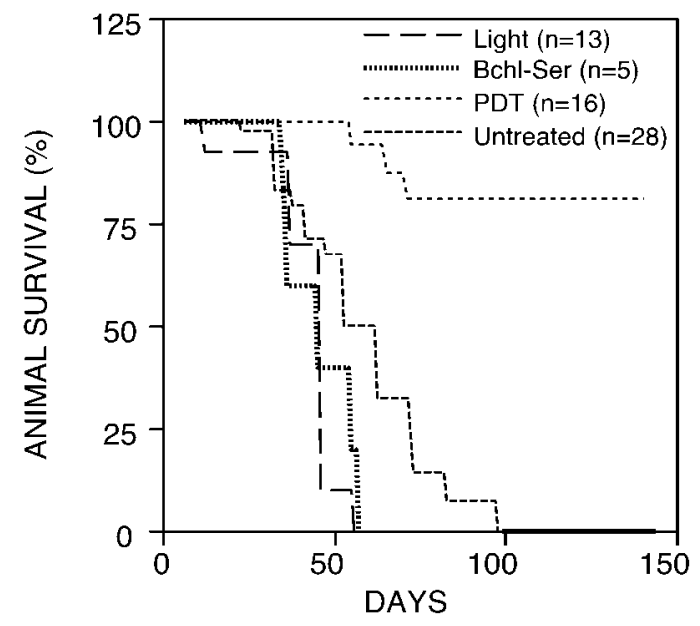

Figure 2. Kaplan Meier survival curves. Survival of CD1 nude mice was followed in four groups: untreated $(\mathrm{n}=28)$, Bchl-Ser-treated but not illuminated $(n=5)$, only illuminated $(n=13)$ and PDTtreated by i.v. injection $(20 \mathrm{mg} / \mathrm{kg} \mathrm{Bchl}-\mathrm{Ser})$ followed by $30 \mathrm{~min}$ illumination at $108 \mathrm{~J} / \mathrm{cm}^{2}(\mathrm{n}=16)$. All other details are as described under "Materials and Methods."

inoculation (Fig. 2). Animals treated with Bchl-Ser but not illuminated (dark controls) had a mean survival time of 43 days. Animals with tumors that were only illuminated (without Bchl-Ser) survived for 40 days. An i.v. injection of 20 $\mathrm{mg} / \mathrm{kg}$ Bchl-Ser immediately followed by a $30 \mathrm{~min}$ illumination at $108 \mathrm{~J} / \mathrm{cm}^{2}$ led to complete healing and eradication of the tumors (4-5 weeks) in 13 out of 16 animals, or an $81 \%$ cure. The cured mice lived their normal life span (814 months) with no signs of tumor recurrence.

The illumination of the tumor always included $2-3 \mathrm{~mm}$ of surrounding normal tissue. Following PDT edema and inflammation became apparent (1-2 days) with development of necrosis and crusting (days 3-5) but only in the tumor region. Edema that healed within a few days leaving no tissue damage was observed in the illuminated normal tissue around the tumor. Some erythema (that cleared within the same time) in the immediately adjacent, unilluminated, normal tissue was invariably observed. In contrast, the healing of the tumor took approximately 4-5 weeks. Occasionally some residual melanin tattoos were observed. The skin covering the tumor was initially damaged but underwent remodeling during the healing process.

In the statistical analysis of this experiment the results of the full treatment (PDT) group (right-censored at 140 days) were compared with each control separately, using two test procedures: the log-rank test and the Wilcoxon test (40). The first test places more weight on longer survival times, while the second places more weight on early survival times. The analyses with both tests gave highly significant results for the comparison with the three control groups, $(P<0.0001)$. The survival curves of the three controls were also compared to see whether they represent similar survival behavior, using the same test procedures. These results showed that the $P$ values were 0.023 and 0.126 , for the $\log$ rank and Wilcoxon tests, respectively. This means that the three controls show similar behavior at early survival times, but differ significantly toward the end. The difference relates mainly to comparison of the light and Bchl-Ser treatment groups with

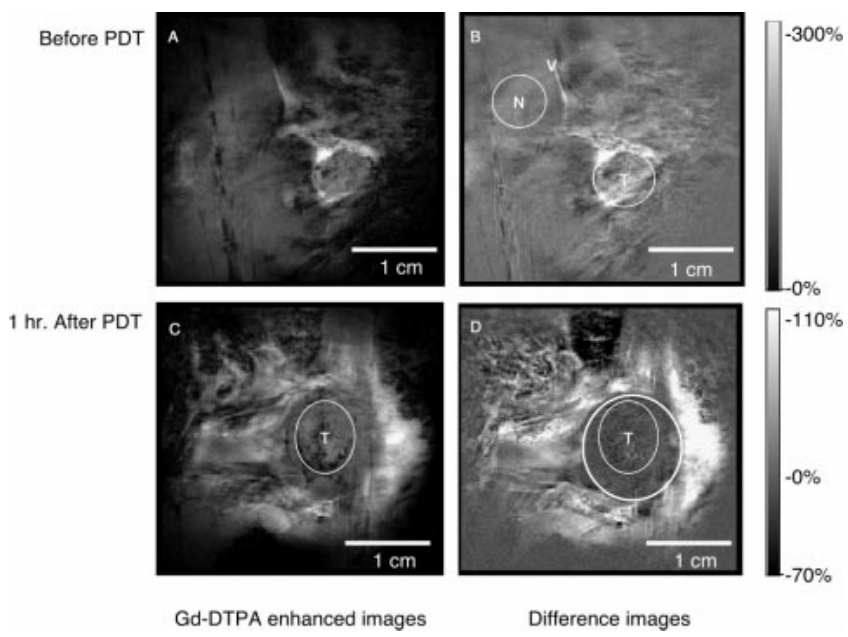

Figure 3. Effect of PDT on tumor vascular permeability as studied by Gd-DTPA-enhanced MRI. PDT (i.v injection of Bchl-Ser, followed by $30 \mathrm{~min}$ illumination) was performed. Gd-DTPA-enhanced images and the difference images were obtained using a $\mathrm{T}_{1}$-weighted gradient echo pulse sequence (TR $30 \mathrm{~ms}$, TE $10.5 \mathrm{~ms}$, slice thickness $1 \mathrm{~mm}$ ). An image taken before Gd-DTPA injection was subtracted from images acquired at given times after Gd-DTPA administration to obtain the difference images. Gd-DTPA-enhanced images and the corresponding difference image before $(\mathrm{A}, \mathrm{B})$ and $1 \mathrm{~h}$ after PD treatment (C, D) are presented. The tumor is marked ' $\mathrm{T}$ '" on the images after PDT. The dark spot seen on the difference image after PDT signifies the reduced signal in the illuminated region (marked by the outer white circle).

the untreated group. For example the $75 \%$ survival quartiles for the Bchl-Ser, light and untreated groups were 35, 35 and 37.5 days, respectively, while the $50 \%$ quartiles were 45,45 and 52.5 days and the $25 \%$ were 50,45 and 65 days, respectively.

\section{MRI study}

Vascular damage caused by PDT was examined by monitoring the uptake of Gd-DTPA using $\mathrm{T}_{1}$-weighted gradient echo images $(\mathrm{n}=6)$ before (Figs. $3 \mathrm{~A}, \mathrm{~B}$ and $4 \mathrm{~A})$, at $1 \mathrm{~h}$ (Fig. 3C,D), and at $24 \mathrm{~h}$ (Fig. 4B) after PDT. The signal enhancement kinetics of Gd-DTPA was determined in three regions of interest (ROI) in the tumor image (Fig. 3B): the entire tumor $(\mathrm{T})$, the adjacent normal tissue $(0.5-1 \mathrm{~cm}$ from the tumor) (N) and a voxel containing a normal blood vessel $(0.8 \mathrm{~cm}$ away from the tumor) $(\mathrm{V})$. The uptake and clearance of Gd-DTPA from each region were determined.

Before PDT uptake and clearance profiles of Gd-DTPA typical for each examined tissues were recorded, indicating vessel intactness and normal perfusion. The percentage of signal enhancement in the indicated ROI (tumor, vessel and normal tissue) are shown (Figs. 3A,B and 4A). One hour after PDT Gd-DTPA was i.v.-injected. The entire illuminated region including the tumor showed signal attenuation, whereas the regions around the illuminated spot showed signal enhancement (Fig. 3D). This result can be explained by the shortening of $\mathrm{T}_{2}$ relaxation due to a massive release of Gd-DTPA in the illuminated zone, presumably because of severe permeability changes of the blood vessels and inflammation in the tumor area. However, the normal tissue regions around the illuminated spot showed signal enhancement but 


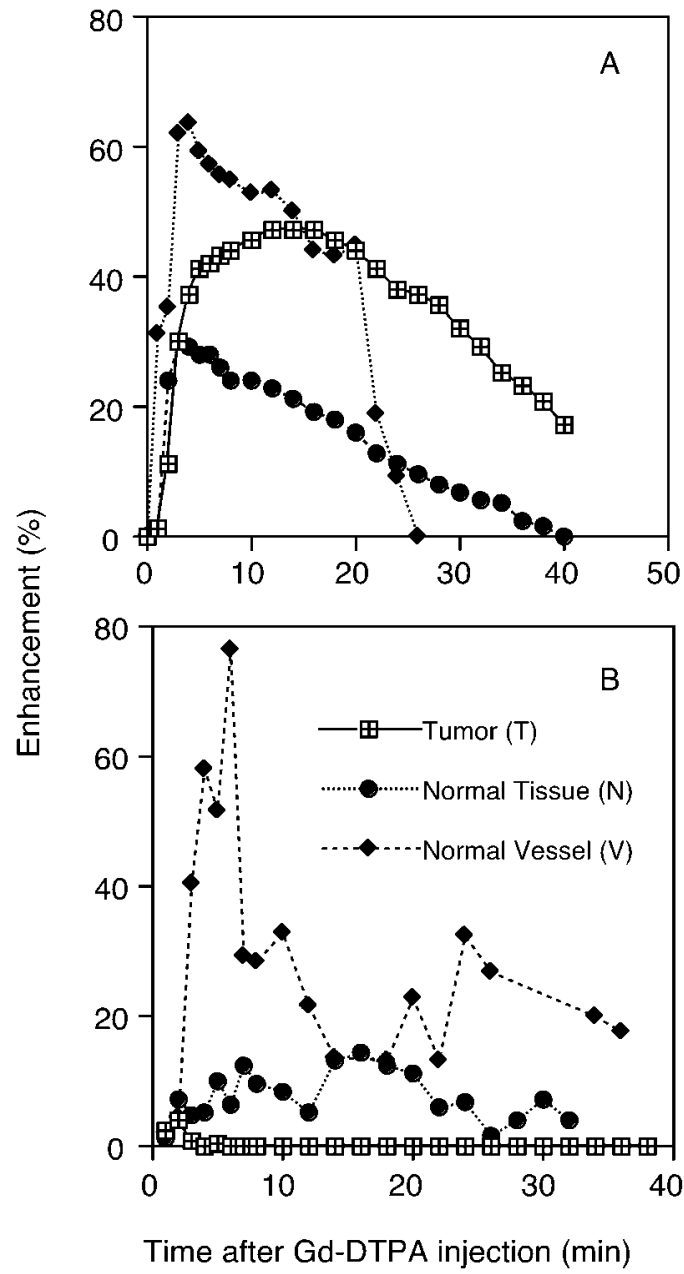

Figure 4. Gd-DTPA enhancement time curves before and $24 \mathrm{~h}$ after PDT. The curves were calculated for the same ROI in Fig. 3B, using the difference images obtained before (A) and $24 \mathrm{~h}$ after PDT (B). Three enhancement time curves in the following ROI were generated from the difference images: the entire tumor $(\mathrm{T})$, the adjacent normal tissue $(0.5-1 \mathrm{~cm}$ from the tumor) $(\mathrm{N})$ and a voxel contained within a normal blood vessel (partial volume of approximately 10\%, 0.8 $\mathrm{cm}$ away from the tumor) $(\mathrm{V})$.

not shortening of $\mathrm{T}_{2}$ values (see also Fig. 5A), presumably due to subtle changes in blood vessel permeability. No such phenomenon was seen before PDT (Fig. 3A,B). Signal enhancement time curves were also derived from the same ROI $24 \mathrm{~h}$ after PDT by using serial $\mathrm{T}_{1}$ images (Fig. 4B). Whereas the perfusion pattern of Gd-DTPA in normal tissue and vessels (as deduced from $\mathrm{T}_{1}$-weighted images) was similar to that observed before PDT (Fig. 4A), the tumor showed a complete lack of tracer uptake ( $0 \%$ enhancement) after PDT (Fig. 4B), suggesting perfusion arrest.

The attenuation in signal intensity in the illuminated area $1 \mathrm{~h}$ after PDT can result from shortening of the $\mathrm{T}_{2}$ relaxation time because of massive accumulation of Gd-DTPA (42). To examine this possibility serial $\mathrm{T}_{2}$-weighted Gd-DTPA enhanced images were taken $1 \mathrm{~h}$ after PDT $(\mathrm{n}=3)($ Fig. 5A). Indeed, we observed substantial attenuation $(\sim-50 \%)$ of signal intensity in the tumor region. The retention of $\mathrm{Gd}-$ DTPA in the PD-treated tumor $20 \mathrm{~min}$ or more after injection of the tracer deviated from the pattern observed in nor-
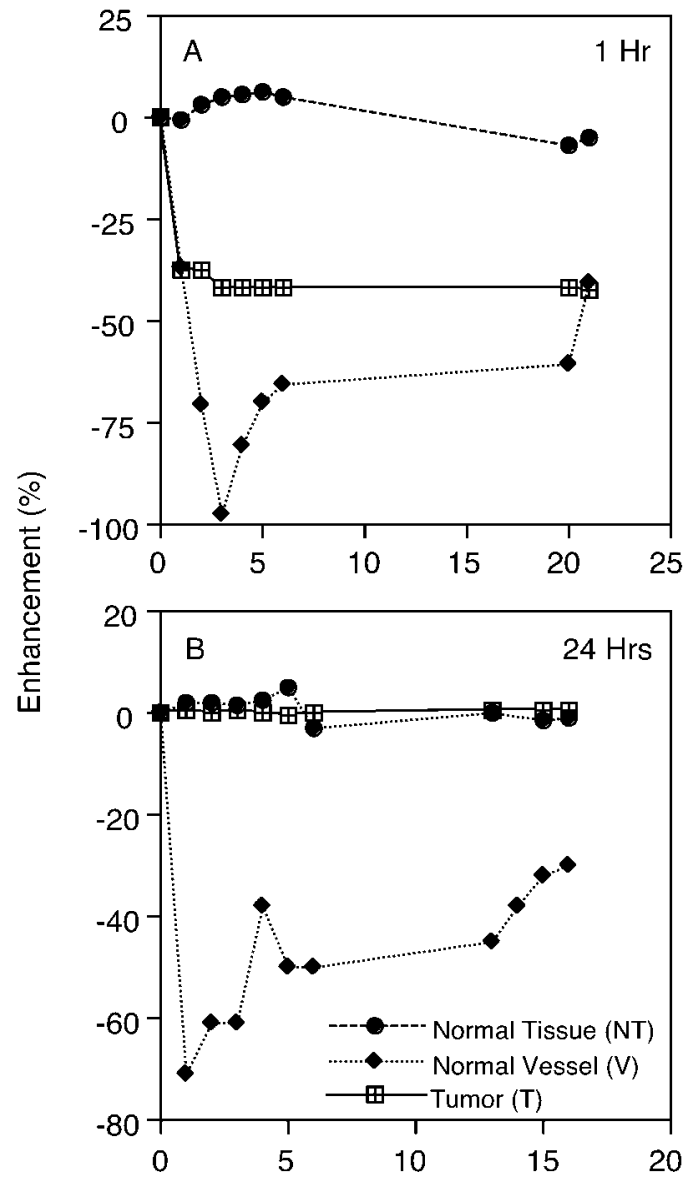

Time after Gd-DTPA injection (min)

Figure 5. Gd-DTPA profile generated from $\mathrm{T}_{2}$-weighted spin echo images 1 and $24 \mathrm{~h}$ after PDT. Serial $\mathrm{T}_{2}$-weighted spin echo images were acquired over a 15 min period after i.v. injection of Gd-DTPA, using segmented RARE of eight echoes per excitation with an effective TE of $77 \mathrm{~ms}$. The field of view was $4 \mathrm{~cm}$ over a $256 \times 128$ matrix, and a slice thickness of $1 \mathrm{~mm}$. The time curves were generated from ROI described in Fig. 3 in the difference images taken $1 \mathrm{~h} \mathrm{(A)} \mathrm{and} 24 \mathrm{~h}$ after PDT (B).

mal tissue and in large vessels of the same animal (Fig. 5A). The Gd-DTPA profile of the $\mathrm{T}_{2}$-weighted images $24 \mathrm{~h}$ after PDT showed that the treated tumor remained completely void of Gd-DTPA, whereas the normal tissue and the vessel behaved as expected (Fig. 5B). This behavior is similar to that observed by a $\mathrm{T}_{1}$-weighted gradient echo pulse sequence (Fig. 4B). In independent experiments, $\mathrm{T}_{2}$-weighted GdDTPA-enhanced images of untreated mice were recorded, and showed reduced signal intensity in the vessel, but not in the tumor domain or in normal tissue. $T_{2}$ maps taken before PDT indicated no change upon administration of Gd-DTPA (Fig. 6A,B). Examination of the contrast in $T_{2}$ maps of the same region confirmed the shortening in basal $T_{2}$ values in the tumor within $1 \mathrm{~h}$ after PDT, even without Gd-DTPA (Fig. 6A,C). This result is consistent with vascular damage and hemorrhage formation. Administration of Gd-DTPA caused a further reduction in $T_{2}$, probably because of massive leakage of the tracer into the tumor (see Fig. 6A-D).

These results suggest that the early vascular damage and enhanced permeability, seen at $60 \mathrm{~min}$, develop into a total 

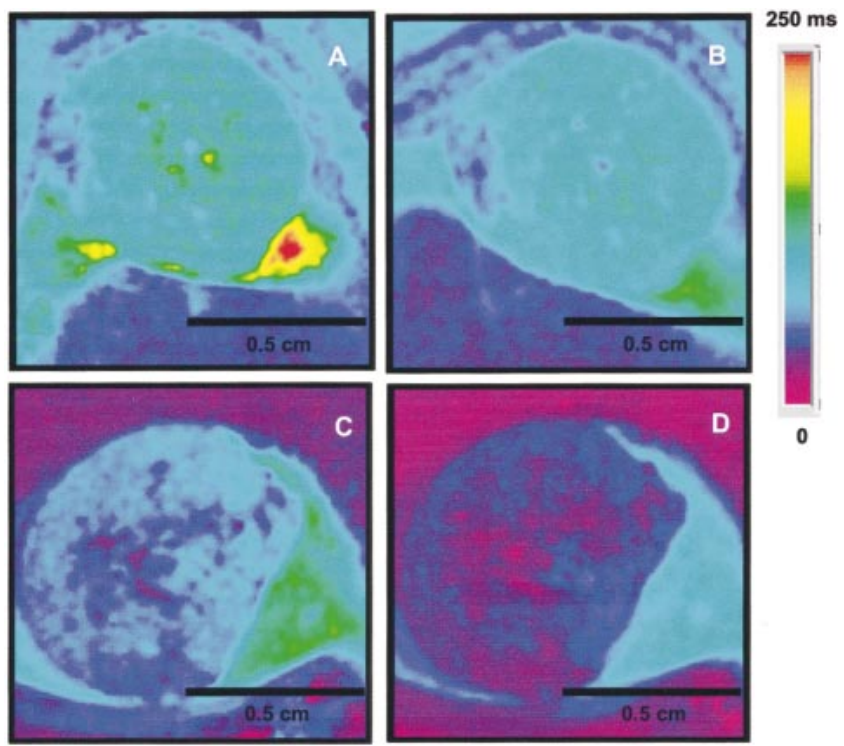

Figure 6. $T_{2}$ maps of the tumor with and without Gd-DTPA before and $1 \mathrm{~h}$ after PDT. $\mathrm{T}_{2}$ maps were generated by single exponential fitting from multiecho $(0-90 \mathrm{~ms})$ pulse sequences with intervals of $15 \mathrm{~ms}$, a time repetition of $1273 \mathrm{~ms}$ and a field of view of $4 \mathrm{~cm}$ over a $256 \times 256$ matrix. The tumor before PDT (A, B) and after PDT $(\mathrm{C}, \mathrm{D})$ is presented. The left and right rows indicate images taken before and 5 min after i.v. injection of Gd-DTPA, respectively. The figure indicates shortening of $\mathrm{T}_{2}$ (mean tumor value) after PDT from 82 to $74 \mathrm{~ms}$ with local values as low as $48 \mathrm{~ms}$ (C) and further shortening (mean tumor value) to $58 \mathrm{~ms}$ with occasional low values of $40 \mathrm{~ms}$ after Gd-DTPA injection (D), as demonstrated by the color scale $\left(\mathrm{T}_{2}, \mathrm{~ms}\right)$.

arrest of tumor perfusion $24 \mathrm{~h}$ after the treatment. To further validate this notion we next examined PDT-induced vascular damage by independent methods.

\section{Determination of vascular leakage of macromolecules}

Using Eu-IgG as a high molecular weight reporter molecule, we observed an increase in vascular permeability after PDT (Table 2). The permeability is expressed as the ratio of EuIgG trapped in the treated as compared to the untreated tumor. More specifically a ratio of 1.0 means no treatment effect. As can be seen PDT increased the ratio of Eu-IgG release into the treated tumors by a factor of $7.1 \pm 2.33$ (n $=6$ ), compared with the contralateral untreated dark controls. Since the ratios do not follow a normal distribution, a one-sided Wilcoxon signed rank test was applied for testing the hypothesis that the median of the ratios is equal 1.0. The obtained result gave $P=0.015$, meaning that the median of the ratios is significantly larger than 1.0. Using the mouse kidneys as bilateral dark controls, we found at the same time that the two kidneys had retained nearly identical Eu-IgG with an average contralateral ratio of $0.94 \pm 0.2$ standard deviations left/right $(\mathrm{n}=6)$. A Wilcoxon signed rank test here gave a $P$-value of 0.56 meaning that the median ratio is not significantly different from 1.0. In light of this we suggest that vascular damage induced by Bchl-Ser-based PDT $1 \mathrm{~h}$ after treatment is severe enough to allow leakage of macromolecules such as $\mathrm{IgG}$ into the tumor interstitial space, a result which complements the MRI analysis.

\section{Histological examination}

Using the classical histological approach, we excised melanoma tumors $24 \mathrm{~h}$ after PDT and examined them by light microscopy after fixation and standard hematoxylin/eosin staining without (Fig. 7A,B) and with light-green staining (Fig. 7C) $(\mathrm{n}=7)$. As illustrated in Fig. 7A, the untreated tumor contains mainly a mass of melanoma cells with occasionally atypical less densely stained picnotic tumor cells in presumed hypoxic regions. The blood vessels (BV) appear intact with a defined clear boundary of endothelial cells. Erythrocytes are seen occasionally within but not outside the vessels. Figure 7B shows clear evidence of massive vascular damage after PDT. The blood vessels became extremely dilated and occluded by thrombi. Hemorrhages characterized by erythrocyte infiltration in certain tumor regions could be observed when the treated tumor slides were stained with light green (Fig. 7C). In some areas, presumably necrotic (NC), the tumor cells appear lighter, picnotic, anucleated and mixed with cell debris. However, after $24 \mathrm{~h}$ a substantial mass of tumor cells remained intact. This microscopic analysis provided further evidence that the vascular bed of the tumor is a primary target of Bchl-Ser-based PDT, as proposed here.

\section{DISCUSSION}

In this study we propose that Bchl-Ser, when used as a photosensitizer in PDT, acts by tumor vascular destruction that results in a high rate of cure in mice. The therapeutic potential of this approach was demonstrated in the successful treatment and cure $(81 \%)$ of melanotic melanoma tumors in

Table 2. Leakage of Eu into PD-treated and-untreated tumors*

\begin{tabular}{|c|c|c|c|c|}
\hline \multirow[b]{2}{*}{ Mouse number } & \multicolumn{2}{|c|}{ Eu-IgG in tumor PDT treated/untreated } & \multicolumn{2}{|c|}{ Eu-IgG in kidney left/right } \\
\hline & Fluorescence (a.u.) & Ratio & Fluorescence (a.u.) & Ratio \\
\hline 1 & $120421 / 20159$ & 5.97 & $82185 / 98459$ & 0.83 \\
\hline 2 & $62722 / 7151$ & 8.77 & $118129 / 133394$ & 0.89 \\
\hline 3 & $127712 / 28125$ & 4.54 & $230746 / 199230$ & 1.16 \\
\hline 4 & $59676 / 5713$ & 10.44 & $108074 / 86023$ & 1.26 \\
\hline 5 & $46266 / 5760$ & 8.03 & $85200 / 108404$ & 0.78 \\
\hline 6 & $89900 / 18020$ & 4.99 & $168269 / 227764$ & 0.74 \\
\hline Ratio, mean $\pm \mathrm{SD}$ & & $7.12 \pm 2.33$ & & $0.94 \pm 0.21$ \\
\hline
\end{tabular}

*Values represent Eu concentrations in fluorescence (arbitrary units) per sample of supernatant obtained from a $100 \mathrm{mg}$ wet weight tissue/ $\mathrm{mL}$ homogenate. 


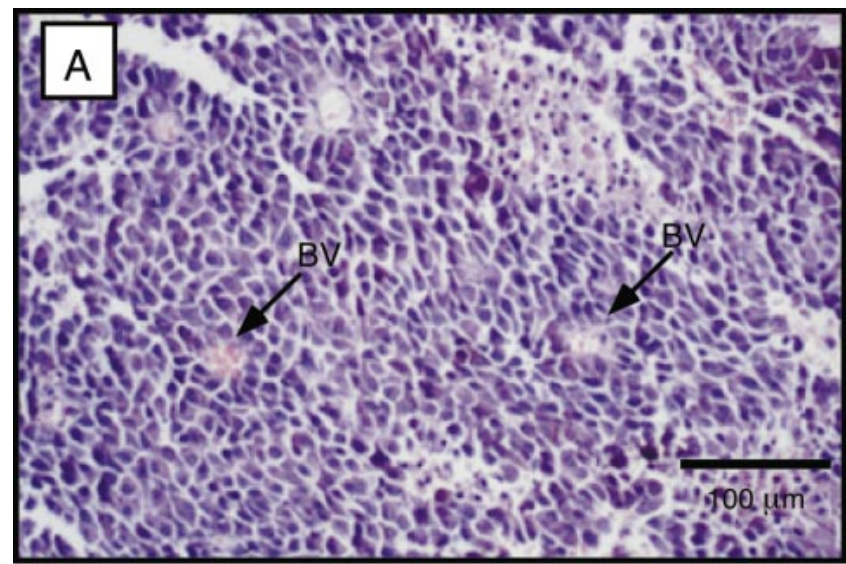

Ser, is based on the following considerations: first, blood oxygen levels are known to be the highest in comparison to surrounding tissues, and sensitizer levels in the blood are maximal shortly after injection (Fig. 1, Table 1), an optimal situation for successful PDT. Second, the vascular endothelial cells and the cellular components of the blood are directly accessible to the blood-borne sensitizer molecules. Third, it is likely that vascular rupture and hemorrhage formation facilitate rapid discharge of the sensitizer molecules into the tumor site more efficiently than expected by passive diffusion across capillary walls. Fourth, use of NIR illumination enables large penetration depth $(9 \mathrm{~mm}$ in $\mathrm{M} 2 \mathrm{R}$ melanoma tumors) (32) and therefore permits the exposure of larger tumor volumes to the light field. Last, tumor-cell killing is presumably achieved by a direct photodynamic insult during illumination and indirectly by subsequent oxygen and nutrient deprivation because of blood stasis that leads to hypoxia (32), necrosis and tumor eradication. These factors are proposed to synergistically contribute to the high success rate of the suggested treatment protocol.

Application of magnetic resonance techniques as well as histological examination sheds light on the primary effects of Bchl-Ser-based PDT and on the integrity of the tumor vasculature. Before PDT, the kinetics of Gd-DTPA uptake and clearance, as exhibited in $\mathrm{T}_{1}$-weighted serial images, was typical for each tissue examined (Figs. 3A,B and 4A). One hour after PDT, however, the kinetic behavior of $\mathrm{T}_{1}$-weighted images in the illuminated region of the tumor changed drastically, with attenuation of the signal resulting from accumulation of Gd-DTPA. This has also been verified in the $\mathrm{T}_{2}$ maps (Fig. 6). Twenty-four hours after PDT we also observed changes (compared to the pre-PDT controls) in the tumor but this time signal intensity remained unchanged, indicating blood stasis (Figs. 4B and 5B). This finding indicates that vascular damage during the treatment already permitted a massive release of the small molecular weight tracer Gd-DTPA into the interstitial volume of the tumor $1 \mathrm{~h}$ later. The degree of damage to the capillaries at this time was extensive enough to allow the release of serum proteins into the tumor, as monitored by the macromolecular probe EuIgG (Table 2). Moreover, the rupture of blood vessels was sufficiently extensive to allow even erythrocytes to infiltrate the tumor tissue. Vascular rupture was further supported by inspection of the tumor MRI contrasts in $\mathrm{T}_{2}$-weighted images (cf Fig. 6A,C). $\mathrm{T}_{2}$ maps obtained $1 \mathrm{~h}$ after the beginning the treatment showed a shortening of tumor mean $\mathrm{T}_{2}$ values from 80 to $74 \mathrm{~ms}$ with much shorter local values of $48 \mathrm{~ms}$. This observation probably reflects localized hemorrhage which increases the content of paramagnetic deoxyhemoglobin. Further shortening of $\mathrm{T}_{2}$ to $58 \mathrm{~ms}$ (mean tumor value) with local values as low as $40 \mathrm{~ms}$ after the injection of $\mathrm{Gd}-$ DTPA were also observed (see Fig. 6B,D), in agreement with the above findings (Fig. 5). The hemorrhagic infiltration of erythrocytes deduced by these MRI images took place within $1 \mathrm{~h}$ after PDT, which probably accounts for the histological observations made $24 \mathrm{~h}$ later (Fig. 7C). In agreement with our interpretation shortening of $T_{2}$ relaxation times in melanotic zones of melanoma xenografts were correlated with spontaneous hemorrhage accompanied by hemoglobin denaturation and the accumulation of paramagnetic ferric iron (45-47). Together, these observations suggest 
that Bchl-Ser-based PDT, as demonstrated here, results in the formation of hemorrhage probably associated with vascular infarction within the first hour of treatment. On the other hand, photofrin II $^{\oplus}$-based PDT of nonmelanotic tumors resulted in an appreciable prolongation of $\mathrm{T}_{1}$ and $\mathrm{T}_{2}$ relaxation times $24 \mathrm{~h}$ after treatment. Moreover, the increased relaxation times in these studies were associated with higher water content or decreased viscosity because of tumor cell lysis (48-51). In an MRI study by Winsborrow et al. (52) maximal effects of PDT with benzoporphyrin derivative (BPD), photofrin and phthalocyanin in M1 murine tumors were obtained when illumination was delayed by 3, 24 and $48 \mathrm{~h}$, respectively, after sensitizer administration. Importantly, no change in the MRI images was observed within 1 $\mathrm{h}$ of illumination for all three sensitizers. Thus, the tumor response was not ascribed to vascular damage and hemorrhage was not observed at times earlier than $24 \mathrm{~h}$ after illumination (52).

The contribution of vascular damage to overall tumor response to PDT has been addressed in several studies (5355). PDT with photofrin in mice led to macromolecular leakage from blood vessels, and to vascular constriction during the illumination. This process developed into blood flow stasis in tumor and muscle tissues (56). In BPD-based PDT acute vascular damage, tumor regression and cure were maximal when the injection-illumination time interval was 5 min and declined thereafter up to $180 \mathrm{~min}$ (19). Our results with Bchl-Ser agree completely with these findings and support the view that vascular stasis plays a significant role in PDTinduced tumor destruction. The success rate of the treatment strategy may be explained in part by a differential response of tumor and adjacent normal tissues. Both tissues respond to illumination with edema; however, the tumor develops severe inflammation, necrosis and tissue remodeling while the normal tissue appears to heal rapidly. This phenomenon will be investigated in greater detail in future studies.

Thus, the responses of tumors to various PDT agents may markedly differ, depending on the nature of the sensitizers and the applied treatment protocols. In Bchl-Ser-based PDT, as suggested here, illumination should begin when maximal sensitizer concentrations in the circulation are attained (Fig. 1, Table 1), whereas sensitizer levels in the tumor tissue are presumably still too low to confer photocytotoxicity. Extension of the injection-illumination time interval reduced PDT efficiency and was therefore minimized (data not shown). In independent experiments performed on cultured cells it was found that at sufficiently high Bchl-Ser concentrations (8$40 \mu \mathrm{g} / \mathrm{mL}$ ), effective PDT can be achieved even without preincubation with the sensitizer (R. Hami, unpublished). Using the proposed PDT protocol described here we attained sensitizer concentrations in this range in the blood causing immediate damage upon illumination. Furthermore, considering the presence of the two photodynamically active degradation products Bphe-Ser and Bchl-Ser-Ox (Mazor, unpublished), the cumulative effective sensitizer concentration in the blood may be even higher $(7-19 \mu \mathrm{g} / \mathrm{mL})$. Under these conditions, the vasculature in the illuminated area becomes the major, if not the only target, for selective photodynamic action. Rapid vascular photosensitization by 2-[1-hydroxyethyl]-2-devinylpyropheophorbide was previously reported (57), but the applicability of this finding to PDT protocols was never proven. Moreover, some reports concerning photofrin $\mathrm{II}^{\oplus}$-based PDT have stated that oxygen deprivation because of vascular damage during illumination reduces treatment efficiency $(2,12,17)$. In contrast the phototoxicity of Bchl-Ser can also be generated under hypoxic conditions (around $2.5 \mathrm{~mm} \mathrm{Hg} \mathrm{O}_{2}$ ), as shown with anaerobic bacteria (58). Since Bchl-Ser is water soluble and strongly absorbs in the NIR the sensitization and subsequent formation of reactive oxygen species is enabled in large areas of the treated tumor. NIR sensitization of Bchl-Ser in aqueous solutions generates mainly hydroxyl radicals, as determined by electron spin resonance and time-resolved spectroscopy, through a mechanism that is still unclear (59). We recently demonstrated the depletion of oxygen during illumination and the development of severe ischemia after Bchl-Ser-based PDT in vivo by using an optical oxygen microsensor (32).

In summary, Bchl-Ser-based PDT offers antivascular tumor therapy that is accomplished concomitantly by sensitizer and light delivery. Whereas antiangiogenic therapies aim at vascular suppression $(8,9)$, the objective of the proposed methodology is to eradicate the tumor by vascular destruction. The high rate of cure achieved with a single half-hour treatment session as reported here for melanotic melanoma suggests that this methodology may be a promising strategy for treating other solid tumors.

Acknowledgements-Y.S. is the incumbent of the Charles and Tillie Lubin Chair of Biochemical Endocrinology. This study was performed in partial fulfillment of J.Z.'s Ph.D. thesis for the Feinberg Graduate School, The Weizmann Institute of Science. We wish to thank Prof. Hadassa Degani for her critical review and helpful discussions, Mr. Steve Mensh for reading the manuscript and Ms. Rina Tzoref for her excellent secretarial assistance. This study was supported in part by grants from the Lynne and William Frankel Fund for the Diagnosis and Treatment of Ovarian and Breast Cancer, the Jaffe Family Foundation, Mrs. S. Zuckerman, Yeda Research and Development Co., Ltd. (Israel), the Israeli Ministry of Science and Technology, the Commission of the European Communities and STEBA BEHEER N.V.

\section{REFERENCES}

1. Stone, H. B., J. M. Brown, T. L. Phillips and R. M. Sutherland (1993) Oxygen in human tumors: correlations between methods of measurement and response to therapy. Radiat. Res. 136, 422434.

2. Dougherty, T. J., C. J. Gomer, B. W. Henderson, G. Jori, D. Kessel, M. Korbelik, J. Moan and Q. Peng (1998) Photodynamic therapy (review). J. Natl. Cancer Inst. 90(12), 889-905.

3. Henning, J. P., R. L. Fournier and J. A. Hampton (1995) A transient mathematical model of oxygen depletion during photodynamic therapy. Radiat. Res. 142, 221-226.

4. Foster, T. H., R. S. Murant, R. G. Bryant, R. S. Knox, S. L. Gibson and R. Hilf (1991) Oxygen consumption and diffusion effects in photodynamic therapy. Radiat. Res. 126, 296-303.

5. Quinn, T. E., G. B. Thurman, A. Sundell, M. Zhang and C. G. Hellerqvist (1995) CM101, a polysaccharide antitumor agent, does not inhibit wound healing in murin models. J. Cancer Res. Clin. Oncol. 121, 253-256.

6. Thorpe, P. E. and F. J. Burrows (1995) Antibody-directed targeting of the vasculature of solid tumors. Breast Cancer Res. Treat. 36, 237-251.

7. Huang, X., G. Molema, S. King, L. Watkins, T. S. Edgington and P. E. Thorpe (1997) Tumor infarction in mice by antibodydirected targeting of tissue factor to tumor vasculature. Science 275, 547-550.

8. O'Reilly, M. S., T. Boehm, Y. Shing, N. Fukai, G. Vasios, W. S. Lane, E. Flynn, J. R. Birkhead, B. R. Olsen and J. Folkman 
(1997) Endostatin: an endogenous inhibitor of angiogenesis and tumor growth. Cell 88(2), 277-285.

9. Boehm, T., J. Folkman, T. Browder and M. S. O'Reilly (1997) Antiangiogenic therapy of experimental cancer does not induce acquired drug resistance. Nature 390, 404-407.

10. Abels, C., R. M. Szeimies, P. Steinbach, C. Richert and A. E. Goetz (1997) Targeting of the tumor microcirculation by photodynamic therapy with a synthetic porphycene. J. Photochem. Photobiol. B: Biol. 40, 305-312.

11. Chaudhuri, K., R. W. Keck and S. H. Selman (1987) Morphological changes of tumor microvasculature following hematoporphyrin derivative sensitized photodynamic therapy. Photochem. Photobiol. 46, 823-827.

12. Davis, R. K., K. Davis, R. C. Straight and M. Waner (1988) Effects of photosensitizer (hematoporphyrin derivative-HPD) and light dose in vascular targets in the albino mouse ear. $\mathrm{La}$ ryngoscope $\mathbf{9 8}, 1-4$.

13. Reed, M. W., F. N. Miller, T. J. Wieman, M. T. Tseng and C. G. Pietsch (1988) The effect of photodynamic therapy on the microcirculation. J. Surg. Res. 45, 452-459.

14. Kennedy, S. D., L. S. Szczepaniak, S. L. Gibson, R. Hilf, T. H. Foster and R. G. Bryant (1994) Quantitative MRI of Gd-DTPA uptake in tumors: response to photodynamic therapy. Magn. Reson. Med. 31(3), 292-301.

15. van Geel, I. P. J., H. Oppelaar, P. F. Rijken, H. J. Bernsen, N. E. Hagemeier, A. J. van der Kogel, R. J. Hodgkiss and F. A. Stewart (1996) Vascular perfusion and hypoxic areas in RIF-1 tumours after photodynamic therapy. Br. J. Cancer 73, 288293

16. Henderson, B. W., A. B. Sumlin, B. L. Owczarczak and T. J. Dougherty (1991) Bacteriochlorophyll-a as photosensitizer for photodynamic treatment of transplantable murine tumors. $J$. Photochem. Photobiol. B: Biol. 10, 303-313.

17. van Geel, I. P. J., H. Oppelaar, Y. G. Oussoren and F. A. Stewart (1994) Changes in perfusion of mouse tumours after photodynamic therapy. Int. J. Cancer 56, 224-228.

18. Fingar, V. H. (1996) Vascular effects of photodynamic therapy. Clin. Laser Med. Surg. 14, 323-328.

19. Fingar, V. H., P. K. Kik, P. S. Haydon, P. B. Cerrito, M. Tseng, E. Abang and T. J. Wieman (1999) Analysis of acute vascular damage after phtodynamic therapy using benzoporphyrin derivative (BPD). Br. J. Cancer 79, 1702-1708.

20. Wu, L. and R. P. Murphy (1999) Photodynamic therapy: a new approach to the treatment of choroidal neovascularization secondary to age-related macular de. Curr. Opin. Ophthalmol. 10, 217-220.

21. Borland, C. F., D. J. McGarvey, A. R. Morgan and T. G. Truscott (1988) Laser flash photolysis of purpurins: novel potential photosensitizers of interest in photodynamic therapy. J. Photochem. Photobiol. B: Biol. 2, 427-434.

22. Morgan, A. R., G. M. Garbo, R. W. Keck, L. D. Kriksen and S. H. Selman (1990) Metallopurpurins and light: effect on transplantable rat bladder tumors and murin skin. Photochem. Photobiol. 51, 589-592.

23. Lee, S.-J. H., N. Jagerovic and K. M. Smith (1993) Use of the chlorophyll derivative, purpurin-18, for syntheses of sensitizers for use in photodynamic therapy. J. Chem. Soc. Perkin Trans. 2369-2377.

24. Nelson, J. S., J. L. McCullough and M. W. Berns (1988) Photodynamic therapy of human malignant melanoma xenografts in athymic nude mice. J. Natl. Cancer Inst. 80, 56-60.

25. Schuitmaker, J. J., J. A. van Best, J. L. van Delft, T. M. Dubbelman, J. A. Oosterhuis and D. de Wolff Rouendaal (1990) Bacteriochlorin a, a new photosensitizer in photodynamic therapy. In vivo results. Investig. Ophthalmol. Vis. Sci. 31, 14441450 .

26. Scherz, A., L. Feodor and Y. Salomon (1997) Chlorophyll and bacteriochlorophyll derivatives, their preparation and pharmacological compositions comprising them. US patent 5,650,292.

27. Rosenbach-Belkin, V., L. Chen, L. Fiedor, F. Tregub, F. Pavlotsky, V. Brumfeld, Y. Salomon and A. Scherz (1996) Serine conjugates of chlorophyll and bacteriochlorophyll: phototoxicity in-vitro and tissue distribution in mice bearing melanoma tumors. Photochem. Photobiol. 64, 174-181.
28. Gross, S., A. Brandis, L. Chen, V. Rosenbach-Belkin, S. Roehrs, A. Scherz and Y. Salomon (1997) Protein A mediated targeting of Bacteriochlorophyll-IgG to Staphylococcus aureus: a model for site specific photocytotoxicity. Photochem. Photobiol. 66 , 872-878.

29. Zilberstein, J., A. Scherz, A. Bromberg, P. Bendel, M. Neeman and Y. Salomon (1994) Chlorophyll based PDT of melanoma: follow up by MRI and by tissue inserted oxygen sensor. In The Tumor Microenvironment: Its Characterization, Modification and Clinical Implication, Faculty of Medicine, University of Granada, Granada, Spain (Abstract).

30. Zilberstein, J., A. Scherz, A. Bromberg, P. Bendel, M. Neeman and Y. Salomon (1995) Mechanisms involved in chlorophyll based photoinduced cell damage: photodynamic therapy of melanoma, Vol. 3, p. 1681. Proceedings of the Society of Magnetic Resonance, Nice, France (Abstract).

31. Zilberstein, J., A. Scherz, A. Bromberg, P. Bendel, M. Neeman and Y. Salomon (1996) The effects of bacteriochlorophyll based PDT on tumor vasculature, Vol. 2, p. 1099. Proceedings of the Society of Magnetic Resonance, New York (Abstract).

32. Zilberstein, J., A. Bromberg, A. Frantz, V. Rosenbach-Belkin, A. Kritzman, R. Pfefermann, Y. Salomon and A. Scherz (1997) Light-dependent oxygen consumption in bacteriochlorophyllserin-treated melanoma tumors: on-line determination using a tissue inserted oxygen microsensor. Photochem. Photobiol. 65, 1012-1019.

33. Zilberstein, J., V. Rosenbach-Belkin, M. Neeman, P. Bendel, F. Kohen, B. Gayer, A. Scherz and Y. Salomon (1998) Bacteriochlorophyll based PDT of solid tumors relies on vascular destruction (Edited by T Patrice). Proceedings of the 7th Biennial Congress, The International Photodynamic Association, ISPEN BIOTECH CD-ROM addition.

34. Scherz, A., Y. Salomon and L. Fiedor (1994) Preparation of chlorophyll conjugates and their application as photosensitizers in photodynamic therapy and general diagnostics. YEDA, Weizmann Institute of Science, European published patent application EP0584552.

35. Gerst, J. E., J. Sole, J. P. Mather and Y. Salomon (1986) Regulation of adenylate cyclase by $\beta$-melanotropin in M2R melanoma cell line. Mol. Cell. Endocrinol. 46, 137-147.

36. DeJordy, J. O., P. Bendel, A. Horowitz, Y. Salomon and H. Degani (1992) Correlation of MR Imaging and histologic findings in mouse melanoma. J. Magn. Reson. Imaging 2, 695-700.

37. Kaplan, E. L. and P. Meier (1958) Nonparametric estimation from incomplete observations. J. Am. Stat. Assoc. 53, 457-481.

38. Barnard, G. and F. Kohen (1990) Idiometric assay: a non-competitive immunoassay for small molecules typified by the measurement of estradiol in serum. Clin. Chem. 36, 1945-1950.

39. Abramovitch, R., G. Meir and M. Neeman (1995) Neovascularization induced growth of implanted C6 glioma multicellular spheroids: magnetic resonance microimaging. Cancer Res. 55, 1956-1962.

40. Kalbfleisch, J. D. and R. L. Prentice (1980) The Statistical Analysis of Failure Time Data. Wiley, New York.

41. Hollander, M. and D. A. Wolfe (1973) Non Parametric Statistical Methods. Wiley, New York.

42. Villringer, A., B. R. Rosen, J. W. Belliveau, J. L. Ackerman, R. B. Lauffer, R. B. Buxton, Y. Chao, V. J. Wedeen and T. J. Brady (1988) Dynamic imaging with lanthanide chelates in normal brain: contrast due to magnetic susceptibility effects. Magn. Reson. Med. 6, 164-174.

43. Lakhani, S., P. Selby and J. M. Blliss (1990) Chemotherapy for malignant melanoma: combination and high doses produce more responses without survival benefit. Br. J. Cancer 61, 330-334.

44. Kollias, N., R. M. Sayre, L. Zeise and M. R. Chedekel (1991) Photoprotection by melanin. J. Photochem. Photobiol. B: Biol. 9, $135-160$.

45. Woodruff, W. J., W. T. Djang, R. E. McLendon, E. R. Heinz and D. R. Voorhees (1987) Intracerebral malignant melanoma: high-field-strength MR imaging. Radiology 165, 209-213.

46. Enochs, W. S., W. B. Hyslop, H. F. Bennett, R. D. Brown, S. H. Koenig and H. M. Swartz (1989) Sources of the increased longitudinal relaxation rates observed in melanotic melanoma. 
An in vitro study of synthetic melanins. Investig. Radiol. 24, 794-804.

47. Rofstad, E. K., E. Steinsland, O. Kaalhus, Y. B. Chang, B. Hovik and H. Lyng (1994) Magnetic resonance imaging of human melanoma xenografts in vivo: proton spin-lattice and spin-spin relaxation times versus fractional tumour water content and fraction of necrotic tumour tissue. Int. J. Radiat. Biol. 65, 387-401.

48. Dodd, N. J., J. V. Moore, D. G. Poppitt and B. Wood (1989) In vivo magnetic resonance imaging of the effects of photodynamic therapy. Br. J. Cancer 60, 164-167.

49. Ceckler, T. L., S. L. Gibson, R. Hilf and R. G. Bryant (1990) In situ assessment of tumor vascularity using fluorine NMR imaging. Magn. Reson. Med. 13, 416-433.

50. Jiang, Q., R. A. Knight, M. Chopp, J. A. Helpern, R. J. Ordidge, Z. X. Qing and F. W. Hetzel (1991) 1H magnetic resonance imaging of normal brain tissue response to photodynamic therapy. Neurosurgery 29, 538-546.

51. Liu, Y. H., R. M. Hawk and S. Ramaprasad (1995) In vivo relaxation time measurements on a murine tumor model-prolongation of T1 after photodynamic therapy. Magn. Reson. Imaging 13, 251-258.

52. Winsborrow, B. G., H. Grondey, H. Savoie, C. A. Fyfe and D. Dolphin (1997) Magnetic resonance imaging evaluation of photodynamic therapy-induced hemorrhagic necrosis in the murine M1 tumor model. Photochem. Photobiol. 66, 847-852.

53. Henderson, B. W. and G. Farrel (1989) Possible implication of vascular damage for tumor cell inactivation in-vivo: comparison of different photosensitizers, Vol. 1065, pp. 2-10. SPIE Proceedings, Photodynamic Therapy: Mechanisms, The International Society for Optical Engineering, Bellingham, WA.

54. Fingar, V. H., T. J. Wieman, S. A. Wiehle and P. B. Cerrito (1992) The role of microvascular damage in photodynamic therapy: the effect of treatment on vessel constriction, permeability, and leukocyte adhesion. Cancer Res. 15, 4914-4921.

55. Henderson, B. W. and T. J. Dougherty (1992) How does photodynamic therapy works? Photochem. Photobiol. 55, 145-157.

56. Fingar, V. H., T. J. Wieman and P. S. Haydon (1997) The effects of trombocytopenia on vessel stasis and macromolecular leakage after photodynamic therapy using photofrin. Photochem. Photobiol. 66(4), 513-517.

57. Bellnier, D. A., Y. K. Ho, R. K. Pandey, J. R. Missert and T. J. Dougherty (1989) Distribution and elimination of Photofrin II in mice. Photochem. Photobiol. 50, 221-228.

58. Chen, L., S. Gross, V. Rosenbach-Belkin, A. Brandis, A. Scherz and Y. Salomon (1998) Photoinactivation of microorganisms by chlorophyll-serin, bacteriochlorophyll-serine and by targeted bacteriochlorophyll-IgG (Edited by T Patrice). Proceedings of the 7th Biennial Congress, The International Photodynamic Association, ISPEN BIOTECH CD-ROM addition.

59. Scherz, A., S. Katz, Y. Vakrat, V. Brumfeld, E. Gabelman, J. Zilberstein, D. Leopold, J. J. Norris, H. Scheer and Y. Salomon (1998) Bacteriochlorophyll-serin based photochemotherapy: type III PDT? In Photosynthsis: Mechanisms and Effects, Vol. V (Edited by G. Garab), pp. 4207-4212. Kluwer, Dordrecht. 